\title{
Constraining Dark Matter lifetime with a deep gamma-ray survey of the Perseus Galaxy Cluster with MAGIC
}

MAGIC Collaboration:, V. A. Acciari ${ }^{\mathrm{a}}$, S. Ansoldi ${ }^{\mathrm{b}, \mathrm{u}}$, L. A. Antonelli ${ }^{\mathrm{c}}$, A. Arbet Engels $^{\mathrm{d}}$, C. Arcaro $^{\mathrm{e}}$,

D. Baack ${ }^{\mathrm{f}}$, A. Babić, ${ }^{\mathrm{g}}$, B. Banerjee ${ }^{\mathrm{h}}$, P. Bangale ${ }^{\mathrm{i}}$, U. Barres de Almeida ${ }^{\mathrm{i}, \mathrm{j}}$, J. A. Barrio ${ }^{\mathrm{k}}$, J. Becerra González $^{\mathrm{a}}$, W. Bednarek ${ }^{1}$, E. Bernardini ${ }^{\mathrm{e}, \mathrm{m}, \mathrm{x}}$, A. Berti ${ }^{\mathrm{b}, \mathrm{y}}$, J. Besenrieder ${ }^{\mathrm{i}}$, W. Bhattacharyya ${ }^{\mathrm{m}}$,

C. Bigongiari ${ }^{\mathrm{c}}$, A. Biland ${ }^{\mathrm{d}}$, O. Blanch ${ }^{\mathrm{n}}$, G. Bonnoli ${ }^{\mathrm{o}}$, R. Carosi ${ }^{\mathrm{p}}$, G. Ceribella ${ }^{\mathrm{i}}$, S. Cikota ${ }^{\mathrm{g}}$, S. M. Colak ${ }^{\mathrm{n}}$,

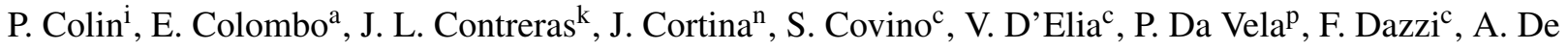
Angelis $^{\mathrm{e}}$, B. De Lotto ${ }^{\mathrm{b}}$, M. Delfino ${ }^{\mathrm{n}, \mathrm{z}}$, J. Delgado $^{\mathrm{n}, \mathrm{z}}$, F. Di Pierro ${ }^{\mathrm{w}}$, E. Do Souto Espiñera ${ }^{\mathrm{n}}$,

A. Domínguez ${ }^{k}$, D. Dominis Prester ${ }^{\mathrm{g}}$, D. Dorner ${ }^{\mathrm{q}}$, M. Doro ${ }^{\mathrm{e}, *}$, S. Einecke $^{\mathrm{f}}$, D. Elsaesser ${ }^{\mathrm{f}}$, V. Fallah

Ramazani $^{\mathrm{r}}$, A. Fattorini ${ }^{\mathrm{f}}$, A. Fernández-Barral ${ }^{\mathrm{e}}$, G. Ferrara ${ }^{\mathrm{c}}$, D. Fidalgo ${ }^{\mathrm{k}}$, L. Foffano ${ }^{\mathrm{e}}$, M. V. Fonseca ${ }^{\mathrm{k}}$,

L. Font ${ }^{\mathrm{s}}$, C. Fruck ${ }^{\mathrm{i}}$, D. Galindo ${ }^{\mathrm{t}}$, S. Gallozzic , R. J. García López ${ }^{\mathrm{a}}$, M. Garczarczyk ${ }^{\mathrm{m}}$, M. Gaug ${ }^{\mathrm{s}}$,

P. Giammariac ${ }^{\mathrm{c}}$, N. Godinovićc ${ }^{\mathrm{g}}$, D. Guberman ${ }^{\mathrm{n}}$, D. Hadasch ${ }^{\mathrm{u}}$, A. Hahn ${ }^{\mathrm{i}}$, T. Hassan ${ }^{\mathrm{n}}$, J. Herrera ${ }^{\mathrm{a}}$, J. Hoang ${ }^{\mathrm{k}}$,

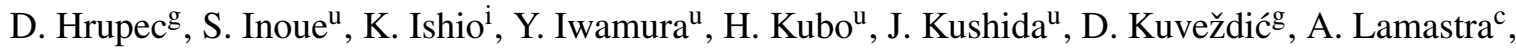

D. Lelas ${ }^{\mathrm{g}}$, F. Leone ${ }^{\mathrm{c}}$, E. Lindfors ${ }^{\mathrm{r}}$, S. Lombardic ${ }^{\mathrm{c}}$, F. Longo ${ }^{\mathrm{b}, \mathrm{y}}$, M. López $^{\mathrm{k}}$, A. López-Oramas ${ }^{\mathrm{a}}$, C. Maggio ${ }^{\mathrm{s}}$,

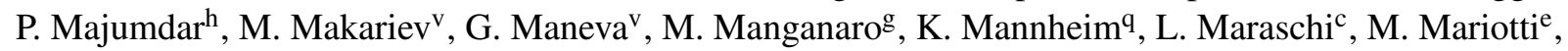

M. Martínez ${ }^{\mathrm{n}}$, S. Masuda ${ }^{\mathrm{u}}$, D. Mazin ${ }^{\mathrm{i}, \mathrm{u}}$, M. Minev ${ }^{\mathrm{v}}$, J. M. Miranda ${ }^{\mathrm{o}}$, R. Mirzoyan ${ }^{\mathrm{i}}$, E. Molinat,

A. Moralejo ${ }^{\mathrm{n}}$, V. Moreno ${ }^{\mathrm{s}}$, E. Moretti ${ }^{\mathrm{n}}$, P. Munar-Adrover ${ }^{\mathrm{s}}$, V. Neustroev ${ }^{\mathrm{r}}$, A. Niedzwiecki ${ }^{1}$, M. Nievas

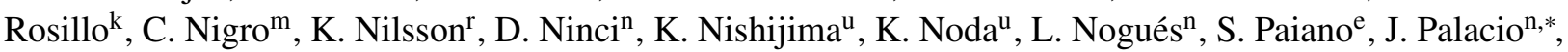

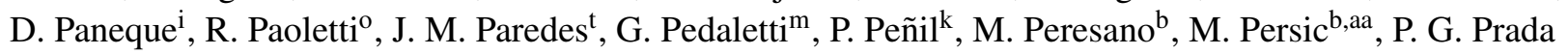
Moroni $^{p}$, E. Prandini ${ }^{\mathrm{e}}$, I. Puljak ${ }^{\mathrm{g}}$, J. R. Garciai , W. Rhode ${ }^{\mathrm{f}}$, M. Ribót ${ }^{\mathrm{t}}$ J. Rico ${ }^{\mathrm{n}, *}$, C. Righi ${ }^{\mathrm{c}}$, A. Rugliancich ${ }^{\mathrm{p}}$ L. Saha ${ }^{k}$, T. Saito ${ }^{u}$, K. Satalecka ${ }^{\mathrm{m}}$, T. Schweizer ${ }^{\mathrm{i}}$, J. Sitarek ${ }^{1}$, I. Šnidarićc ${ }^{\mathrm{g}}$, D. Sobczynska ${ }^{1}$, A. Somero ${ }^{\mathrm{a}}$,

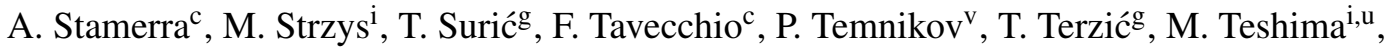

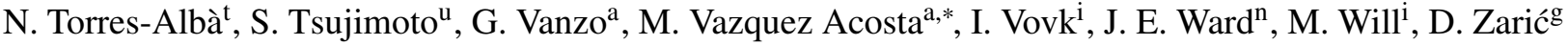

${ }^{a}$ Inst. de Astrofísica de Canarias, E-38200 La Laguna, and Universidad de La Laguna, Dpto. Astrofísica, E-38206 La Laguna, Tenerife, Spain

${ }^{b}$ Università di Udine, and INFN Trieste, I-33100 Udine, Italy

${ }^{c}$ National Institute for Astrophysics (INAF), I-00136 Rome, Italy ${ }^{d}$ ETH Zurich, CH-8093 Zurich, Switzerland

${ }^{e}$ Università di Padova and INFN, I-35131 Padova, Italy

${ }^{f}$ Technische Universität Dortmund, D-44221 Dortmund, Germany

${ }^{g}$ Croatian MAGIC Consortium: University of Rijeka, 51000 Rijeka, University of Split - FESB, 21000 Split, University of Zagreb FER, 10000 Zagreb, University of Osijek, 31000 Osijek and Rudjer Boskovic Institute, 10000 Zagreb, Croatia.

${ }^{h}$ Saha Institute of Nuclear Physics, HBNI, 1/AF Bidhannagar, Salt Lake, Sector-1, Kolkata 700064, India ${ }^{i}$ Max-Planck-Institut für Physik, D-80805 München, Germany

${ }^{j}$ now at Centro Brasileiro de Pesquisas Físicas (CBPF), 22290-180 URCA, Rio de Janeiro (RJ), Brasil

${ }^{k}$ Unidad de Partículas y Cosmología (UPARCOS), Universidad Complutense, E-28040 Madrid, Spain

${ }^{l}$ University of Łódź, Department of Astrophysics, PL-90236 Łódź, Poland

${ }^{m}$ Deutsches Elektronen-Synchrotron (DESY), D-15738 Zeuthen, Germany

${ }^{n}$ Institut de Física d'Altes Energies (IFAE), The Barcelona Institute of Science and Technology (BIST), E-08193 Bellaterra (Barcelona), Spain

${ }^{\circ}$ Università di Siena and INFN Pisa, I-53100 Siena, Italy

${ }^{p}$ Università di Pisa, and INFN Pisa, I-56126 Pisa, Italy

${ }^{q}$ Universität Würzburg, D-97074 Würzburg, Germany

${ }^{r}$ Finnish MAGIC Consortium: Tuorla Observatory and Finnish Centre of Astronomy with ESO (FINCA), University of Turku, Vaisalantie 20, FI-21500 Piikkiö, Astronomy Division, University of Oulu, FIN-90014 University of Oulu, Finland ${ }^{5}$ Departament de Física, and CERES-IEEC, Universitat Autònoma de Barcelona, E-08193 Bellaterra, Spain

${ }^{t}$ Universitat de Barcelona, ICCUB, IEEC-UB, E-08028 Barcelona, Spain 


\begin{abstract}
Clusters of galaxies are the largest known gravitationally bound structures in the Universe, with masses around $10^{15} \mathrm{M}_{\odot}$, most of it in the form of dark matter. The ground-based Imaging Atmospheric Cherenkov Telescope MAGIC made a deep survey of the Perseus cluster of galaxies using almost $400 \mathrm{~h}$ of data recorded between 2009 and 2017. This is the deepest observational campaign so far on a cluster of galaxies in the very high energy range. We search for gamma-ray signals from dark matter particles in the mass range between $200 \mathrm{GeV}$ and $200 \mathrm{TeV}$ decaying into standard model pairs. We apply an analysis optimized for the spectral and morphological features expected from dark matter decays and find no evidence of decaying dark matter. From this, we conclude that dark matter particles have a decay lifetime longer than $\sim 10^{26} \mathrm{~s}$ in all considered channels. Our results improve previous lower limits found by MAGIC and represent the strongest limits on decaying dark matter particles from ground-based gamma-ray instruments.
\end{abstract}

Keywords: decaying dark matter, cluster of galaxies, indirect searches, Imaging Air Cherenkov Telescopes, Perseus

\title{
1. Introduction
}

Decades of observational evidence show that the Standard Model (SM) of Particles Physics cannot entirely explain the gravitational balance observed at all cosmological scales, from that of Milky Way satellite dwarf spheroidal galaxies (dSphs) to that of cluster of galaxies (CGs, see Roos, 2010; Freese, 2009). In order to explain these observations, Dark Matter (DM) has been suggested to exist in the form of a new elementary particle, currently only seen through its gravitational imprint. Weakly-Interacting Massive Particles (WIMPs) are generic massive particles with an expected mass range between few GeV (Lee-Winberg limit, see Boehm et al., 2004) and few hundreds of TeV (unitary bound, see Griest and Kamionkowski, 1990). WIMPs are expected to interact with SM particles with strengths at the weak scale, and to be either stable or very long lived. A WIMP can either annihilate or decay into SM particles, or even be decoupled from the SM. The WIMP paradigm has been long debated, as the WIMP self-annihilation in the early Universe naturally accounts for the DM density observed at present (typically referred to as the WIMP miracle), being possibly within reach of different currently operating instruments. The case of DM annihilation has received greater attention in the literature (Feng, 2010) but there is no experimental or theoretical guarantee that DM particles are absolutely stable. The only constraint is that decaying DM particles' lifetime should be comparable or larger than the Hubble time of $\sim 10^{17} \mathrm{~s}$ in order to explain the current DM density. Among others,

*Corresponding authors: jpalacio@ifae.es, michele.doro@unipd.it, monicava@iac.es, jrico@ifae.es 
decaying DM particles may produce e.g. leptons, quarks, or gauge bosons, which can subsequently provide electromagnetic radiation due to prompt emission or secondary interactions. Lately, DM models that favour decays into leptons (known as "leptophilic" models) have received increased attention, due to the excess of positron events observed in the local cosmic ray (CR) flux by PAMELA, AMS-II and Fermi-LAT (Adriani et al., 2009, Abdo et al., 2009; Ackermann et al., 2010;, Aguilar et al., 2013).

The standard cosmological model predicts CGs to be the latest and most massive structures to form in the Universe (Peebles, 1994). With higher DM concentration and closer distances, dSphs and the Galactic Center are among the best regions to search for annihilating WIMPs. CGs however, with masses of the order of $10^{14-15} \mathrm{M}_{\odot}(\sim 80 \%$ of it in the form of DM, see e.g., Jeltema et al. 2009; Pinzke et al., 2009), are excellent laboratories to study decaying DM. The Perseus CG is a cool-core cluster located at a distance of $77.7 \mathrm{Mpc}$ (redshift $z=0.0183$ ). Perseus is very bright in X-rays, and one of the best candidates for detecting CR induced gamma rays that come from particle acceleration at the cluster core (Aleksić et al., 2010a; Pinzke and Pfrommer, 2010, Pinzke et al., 2011). The Perseus CG is considered among the most promising CGs for gamma-ray indirect DM detection (Sánchez-Conde et al., 2011).

The Major Atmospheric Gamma Imaging Cherenkov (MAGIC) telescopes (Aleksić et al., 2016a) have observed the Perseus CG since 2009, the deepest exposure the instrument has carried out. The campaign took place over several consecutive years and comprised almost $400 \mathrm{~h}$ of recorded data until 2017. MAGIC is a system of two $17 \mathrm{~m}$ diameter Imaging Atmospheric Cherenkov Telescopes (IACTs) capable of detecting gamma rays in the very high energy (VHE, E $>50 \mathrm{GeV}$ ) band. For low zenith angle observations, MAGIC has an angular resolution of $\sim 0.1^{\circ}$, a trigger threshold of $\sim 50 \mathrm{GeV}$, and sensitivity for point-like sources of $\sim 0.66 \%$ of Crab Nebula flux above $220 \mathrm{GeV}$ in $50 \mathrm{~h}$ of observation (Aleksić et al., 2016a). The MAGIC campaign on Perseus CG proved to be very fruitful, producing the strongest limits on CR acceleration and CR pressure in the core of the cluster (Aleksić et al., 2010a, 2012c); a clear detection and model for the radio galaxy NGC 1275, at the center of the cluster (Aleksić et al., 2012d, 2014b); and the detection of the peculiar radio galaxy IC 310, located at $0.6 \mathrm{deg}$ from the Perseus CG center, which provides important evidence related to the acceleration of CRs close to black holes (Aleksić et al., 2010b, 2014a d).

In this work we focus on the search for signatures of decaying DM in an extended region from the Perseus CG with observations from MAGIC. We do not consider the annihilation case since the expected signal of DM annihilation in the Perseus CG would be one order of magnitude smaller than the signal expected for the typical case of dSphs (Sánchez-Conde et al. 2011). More importantly, the expected morphology of the signal of annihilating DM is more concentrated towards the center of the cluster that of decay where, in the case of the Perseus CG, we have limited sensitivity due to the presence of the variable flux gamma-ray emitter NGC 1275. We search for signatures of decaying DM particles in the mass range between $200 \mathrm{GeV}$ and $200 \mathrm{TeV}$ for hadronic/leptonic decays, and for DM particles with masses between $200 \mathrm{GeV}$ and $20 \mathrm{TeV}$ decaying into $\gamma \gamma$. We find no evidence for a DM signal and consequently set $95 \%$ confidence level (CL) lower-limits on the decay lifetime of the DM particle for the different assumed mass values and decay channels.

The rest of this paper is structured as follows. Section 2 discusses the expected DM distribution of the Perseus CG and the photon flux at Earth coming from DM decays. Section 3 describes the MAGIC observations and event selection, optimized to search for DM decays. In Section 4 we introduce the likelihood formalism used in the analysis. In Section 5 we present the obtained lower limits on the decay lifetime, which are put into context with other measurements. In Section 6 the paper is briefly summarized and the 
conclusions are given.

\section{Expected Dark Matter Decay Signal from Perseus}

In order to compute the gamma-ray rate expectations and tailor the data reconstruction and analysis, we first need to assess the DM framework. The differential gamma-ray flux coming from decaying DM from a given direction in the sky is given by:

$$
\frac{\mathrm{d}^{2} \Phi}{\mathrm{d} E \mathrm{~d} \Omega}=\frac{1}{4 \pi} \frac{1}{\tau_{\mathrm{DM}} m_{\mathrm{DM}}} \frac{\mathrm{d} N_{\gamma}}{\mathrm{d} E} \frac{\mathrm{d} J_{\mathrm{dec}}}{\mathrm{d} \Omega}
$$

where $m_{\mathrm{DM}}$ is the DM mass, $\tau_{\mathrm{DM}}$ the DM particle lifetime, $\mathrm{d} N_{\gamma} / \mathrm{d} E$ is the average decay spectrum per reaction, and $\mathrm{d} J_{\mathrm{dec}} / \mathrm{d} \Omega$ is called the differential astrophysical factor (or simply differential $J$-factor, Bergstrom et al. 1998) and is obtained integrating the DM density $\rho$ over the line-of-sight (l.o.s.) for the decay reaction:

$$
\frac{\mathrm{d} J_{\mathrm{dec}}}{\mathrm{d} \Omega}=\int_{\text {l.o.s. }} \mathrm{d} l \rho(l, \Omega) .
$$

The total $J$-factor enclosed in a given sky region can be obtained integrating Equation 2 over a solid angle $\Delta \Omega$. We proceed now in discussing the terms of Equation 1 separately.

\subsection{Decay DM particle models}

An important constraint for decaying DM comes from the fact that, if DM particles were in thermal equilibrium in the early Universe, $\tau_{\mathrm{DM}}$ should be larger than the age of the Universe in order to explain the current observed DM density. Scenarios with such candidates should only allow for a small violation of their stability (Berezinsky et al., 1991; Chen and Kamionkowski, 2004, Ando and Ishiwata, 2015). Alternatively, the stability can be related to the strength of the space-time curvature enabling the so-called gravity portal (Catá) et al. 2017). There are several valid candidates for decaying DM proposed in the literature (see, e.g., Feng, 2010), such as the sterile neutrino, the axion, or the super-symmetric candidates gravitino, lightest righthanded sneutrino, and wino. Regardless the microscopic nature of the particle, DM decays can be roughly classified as (i) leptonic, (ii) hadronic, or (iii) a mix of the two, according to the particle predominance in the decay products. DM candidates with masses at the TeV scale and leptophilic decay modes have been proposed in order to match the measured spectral features seen in CR positron data (see e.g. Feng et al., 2014).

Additionally, decay processes may also give rise to monochromatic photon lines (Garny et al., 2011). Among the candidates previously mentioned, the sterile-neutrino (Ando and Kusenko, 2010) or the gravitino with masses below 2-300 GeV (Ibarra, 2012) could produce such a clear signal that hardly any astrophysical process can mimic.

\subsection{Dark Matter Distribution}

The DM density profile of different astrophysical sources (e.g., the Galactic Center, dSphs and CGs) is generally considered universal and can be expressed by a Zhao-Hernquist functional form (Hernquist, 1990, Zhao, 1996) as

$$
\rho(r)=\frac{\rho_{0}}{\left(\frac{r}{r_{s}}\right)^{\gamma}\left[1+\left(\frac{r}{r_{s}}\right)^{\alpha}\right]^{(\beta-\gamma) / \alpha}},
$$




\begin{tabular}{|c|c|c|c|c|c|c|c|}
\hline \multirow[b]{5}{*}{ Period } & \multirow[b]{5}{*}{ Dates } & & & & & & \\
\hline & & \multicolumn{6}{|c|}{ Telescope Pointing } \\
\hline & & \multicolumn{3}{|c|}{$\mathcal{A}$} & \multicolumn{3}{|c|}{$\mathcal{B}$} \\
\hline & & \multirow[b]{2}{*}{ All data $[\mathrm{h}]$} & \multicolumn{2}{|c|}{ Data Selection } & \multirow[b]{2}{*}{ All data $[\mathrm{h}]$} & \multicolumn{2}{|c|}{ Data Selection } \\
\hline & & & quality [h] & $\begin{array}{c}\text { quality + } \\
\text { specific [h] }\end{array}$ & & quality [h] & $\begin{array}{c}\text { quality + } \\
\text { specific [h] }\end{array}$ \\
\hline $\mathcal{P} 1$ & $2009.11 .01-2011.06 .01$ & 94.7 & 56.4 & 45.4 & - & - & - \\
\hline $\mathcal{P} 2$ & 2012.09.01-2013.01.17 & 9.2 & 9.1 & 9.1 & 59.4 & 40.2 & 36.8 \\
\hline $\mathcal{P} 3$ & 2013.07.27-2014.08.05 & 17.5 & 16.7 & 14.8 & 55 & 30.2 & 28.9 \\
\hline $\mathcal{P} 4$ & 2014.08.31-2014.11.22 & 16.6 & 10.4 & 10.1 & 21.7 & 21.7 & 7.5 \\
\hline $\mathcal{P 5}$ & 2014.11.24-2016.04.28 & 6.8 & 3.9 & 3.9 & 29.3 & 22.32 & 21.9 \\
\hline $\mathcal{P} 6$ & 2016.04.29-2017.08.02 & 44.1 & 41.9 & 12.2 & 20.5 & 16.02 & 11.1 \\
\hline & TOTAL & 185.9 & 138.4 & 106.1 & 188.9 & 119.2 & 96.2 \\
\hline
\end{tabular}

Table 1: Observations of the Perseus cluster with the MAGIC telescopes for two different telescope pointings $\mathcal{A}$ and $\mathcal{B}$ for different observational periods. The number of hours taken for each period and after data selection: quality cuts are based on NSB and aerosol extinction, specific cuts are based on the night-wise significance of $N G C 1275, N G C 1265$ or $I C 310$ (see text for details).

where $r$ is the distance from the DM dynamical center of the cluster, $r_{s}$ and $\rho_{0}$ are the characteristic scale radius and DM density, and $\alpha, \beta, \gamma$ are free parameters. Due to hierarchical structure formation, the total DM profile expressed in Equation 2 is the sum of a smooth component and a second component due to a large expected number of small DM substructures. The effect of DM substructures in the case of DM annihilation in CGs can increase the total astrophysical factor for annihilation $J_{\text {ann }}$ by a factor up to a few tens (Sánchez-Conde et al., 2011; Moliné et al., 2017). In the case of decaying DM however, because of the linear dependence with the DM density (see Equation 2), substructures tend to average out for large observation angles and do not have a sizeable effect on $J_{\mathrm{dec}}$.

We follow the prescription in Sánchez-Conde et al. (2011), where the DM density profile of the Perseus CG is modelled with a Navarro-Frenk-White parametrization (e.g. a Zhao-Hernquist profile with $\alpha=1$, $\beta=3$ and $\gamma=1$, Navarro et al., 1996) with $r_{s}=0.477 \mathrm{Mpc}$ and $\rho_{0}=7.25 \times 10^{14} \mathrm{M}_{\odot} \mathrm{Mpc}^{-3}$. In our analysis we consider the entire DM halo of the Perseus cluster (with a radius of $\sim 1.5^{\circ}$ ), which results in total decay $J$-factor of $1.5 \times 10^{19} \mathrm{GeV} \mathrm{cm}^{-2}$. During the analysis, further angular cuts are applied, which will effectively reduce that value (see Section 3.2).

The estimation of $J_{\mathrm{dec}}$ is proportional to the total DM mass in the source, and hence this is the largest source of uncertainty. In order to be considered in our analysis, these uncertainty should be known as a function of the integration angle ( $\Delta \Omega$ in Equation 2), which is not the case. Mass estimates for CGs show agreement of the order of $4 \%$ uncertainty between lensing and hydrostatic estimation on a sample of 50 CGs (Smith et al., 2016). However, the Perseus CG was not included in this study likely due to its vicinity or ample extension in the sky. For this reason, current available measurements of the total mass of the Perseus CG (Reiprich and Boehringer, 2000; Chen et al., 2007) have larger associated uncertainties of about 30\%. No uncertainties in the $J$-factor are considered in our analysis, but even assuming a $50 \%$ uncertainty on $J_{\mathrm{dec}}$, our lower limits on the lifetime would be weakened by only a factor 2 .

\section{MAGIC observation and data selection}

MAGIC observations were carried out in wobble mode (Fomin et al., 1994), where both the signal (ON) and background control (OFF) regions are observed within the same field of view (FoV). The data were taken in 


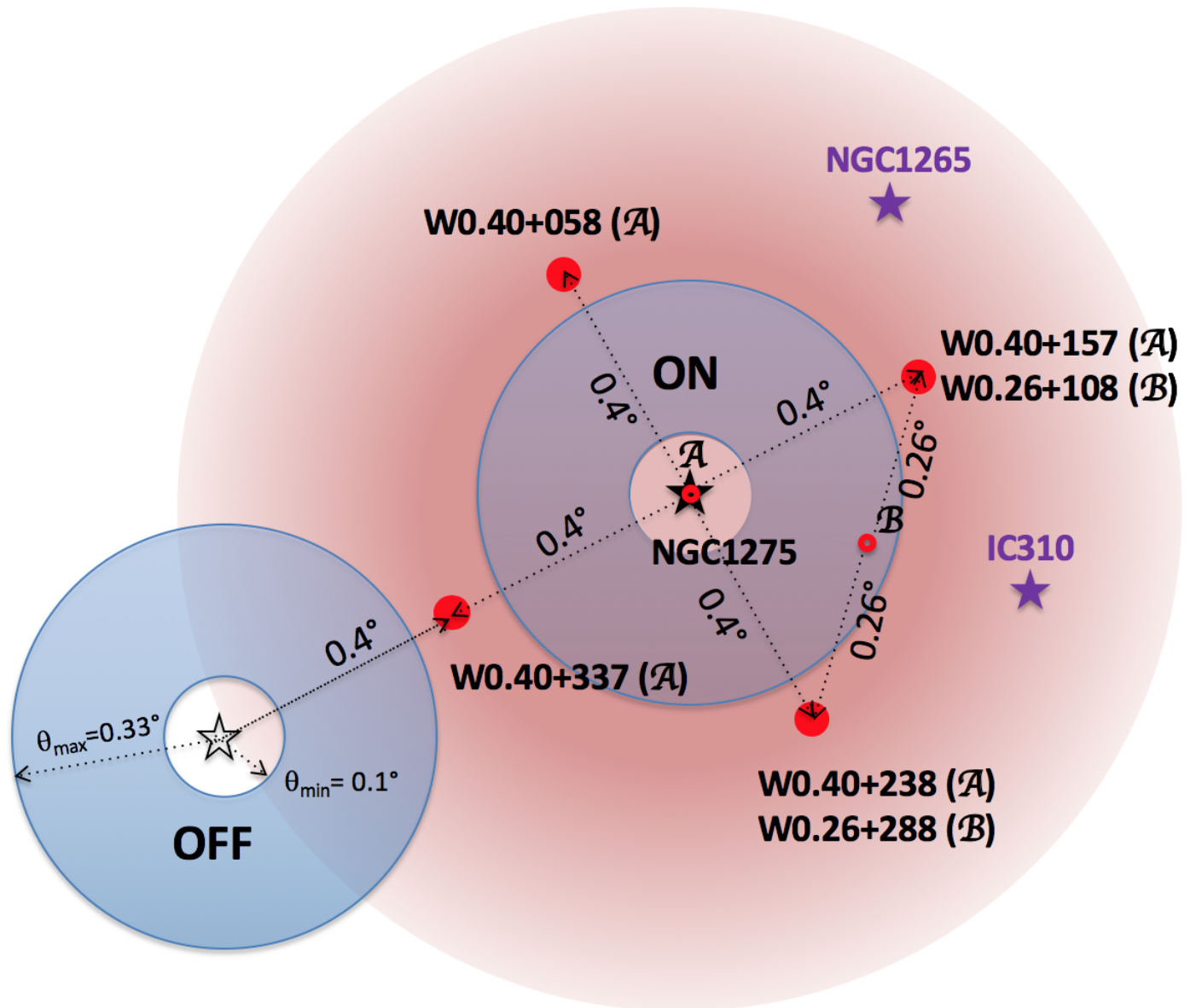

Figure 1: Schematic view of the Perseus CG FoV. The location of the galaxies NCG 1275, IC 310, and NCG 1265 are marked with colored stars (the location of NCG 1275 is coincident with the center of the Perseus CG). The large blurred red region represents the expected DM decay signal morphology (based on Sánchez-Conde et al. 2011). The nominal position of the two pointing modes labeled $\mathcal{A}$ and $\mathcal{B}$ are shown as open red circles whereas the different pointing positions of the telescopes around these two pointing mode directions are labeled W0.40+XXX and W0.26+YYY (for pointing mode $\mathcal{A}$ and $\mathcal{B}$ respectively) and are shown as red wide dots. ON/OFF regions from opposing pointings (e.g. ON from W0.40+157 and OFF from W0.40+337, where OFF center position is marked with an empty star) are analyzed in pairs. $\mathcal{R} 1$ is the region around NCG 1275 defined by $\theta<\theta_{\min }$ (shown with dashed black arrows only for OFF) with respect to NGC 1275's direction. $\mathcal{R} 2$ is the region defined between $\theta_{\min }<\theta<\theta_{\max }$ and are shown as blue regions for ON and OFF. Dark matter is searched within $\mathcal{R} 2$ while $\mathcal{R} 1$ is used to evaluate the gamma-ray emission activity of NGC 1275 for each given dataset.

two different observation pointing modes (here labelled $\mathcal{A}$ and $\mathcal{B}$ ). The gamma-ray emitting radio-galaxy NGC 1275 is located at the dynamical center of the cluster (see Figure 1), and for observation mode $\mathcal{A}$, four symmetric pointing positions are taken at $0.4^{\circ}$ distance around this point. In pointing mode $\mathcal{B}$, the instrument wobbles around a point half-distance between NGC 1275 and IC 310 (pointing alternately in two of the pointing positions of mode $\mathcal{A}$ ). The galaxy NGC 1265 is another important object in the FoV. NGC 1265 is clearly visible in X-rays (Sun et al., 2005) and, albeit never detected above $E>1 \mathrm{GeV}$, is treated as a potential gamma-ray emitter in the analysis.

During the observation campaign, the MAGIC telescopes underwent several hardware upgrades (Aleksić 
et al. 2016a b), leading to six different hardware stable periods (from $\mathcal{P} 1$ to $\mathcal{P} 6$ in Table 1). Appropriate Monte Carlo (MC) simulations for each period are generated to determine the corresponding instrument response function (IRF i.e., the effective area for signal, the angular resolution and bias of the energy reconstruction).

For each data sample, the standard MAGIC event reconstruction (Aleksić et al., 2012b) is applied. Data selection is performed in two different steps, first based on quality cuts and secondly on specific cuts (see Table 1 for details on the amount of data surviving each data selection criteria). Quality cuts are used to select data runs of $\sim 20$ minutes duration with the zenith angle ranging between $5^{\circ}$ and $50^{\circ}$. Only a minor fraction of the data recorded was taken with zenith angles above $50^{\circ}$. A second quality cut was based on the intensity of the night sky background (NSB) that, if too large, also significantly reduces the performance. We allowed the average camera illumination to be no larger than three times that of a standard dark night (as suggested in Ahnen et al., 2017). Furthermore, we selected data based on atmospheric transparency measured with the MAGIC LIDAR instrument (Fruck et al., 2014), requiring a atmospheric optical depth in the direction of the telescope pointing larger than $85 \%$ that of a clear night (which guarantees acceptable performance and systematics below those quoted in Aleksić et al., 2016a). Finally, an event-wise cut based on the size of the event (the total integrated charge contained in a shower image) of 80 photo-electrons is applied. This is slightly higher than the one used for standard low zenith observations to compensate for the larger extinction of Cherenkov light from events at higher zenith values present in our data.

In a second step, specific cuts are used to remove observation nights in which the detection significance (defined in Li and Ma, 1983) of any of the astrophysical sources NCG 1275, NGC 1265, and IC 310 (colored star markers in Figure 1) is higher than $3 \sigma$. The gamma-ray emission of these sources may vary from night to night both in intensity and spectral morphology. Since we search for a steady signal of DM, excluding from the search data from these nights minimizes possible systematic effects introduced by astrophysical signal contamination. No bias is introduced in the search for DM since the evaluation of the detection significance of NGC 1275, NGC 1265 and IC 310 is performed out of the signal region used for the decaying DM search.

Finally, events surviving all the aforementioned data selection criteria are assigned an estimated energy and direction, and a parameter called "hadronness" or $h$ (based on a random forest method, as explain in Albert et al., 2008), which estimates the hadron or gamma-ray origin of an event.

\subsection{Dark Matter signal region}

For an accurate computation of the IRF of the analysis, the morphology of the expected DM signal (described in Section 2] is used to tune the distribution of simulated MC events. This procedure was first applied by Ahnen et al. (2018) during the DM search from the Ursa Major II dSph and was discussed extensively in Palacio (2018). Moreover, in order to avoid contamination from gamma rays coming from NGC 1275, we construct a ring-shaped signal-search region $\mathcal{R} 2$ (defined by two angular distances $\theta_{\min }$ and $\theta_{\max }$ described in Figure 1. Apart from excluding the location of NGC 1275 from the region of interest $\mathcal{R} 2$, its astrophysical contamination inside $\mathcal{R} 2$ due to miss-reconstructed events is also estimated and included in the analysis. Due to the large extension of the decay DM signal and the finite distance between ON and OFF regions (regions around black and empty stars in Figure 1), OFF regions are not fully signal-free (in other words, DM events are expected inside the OFF region for all pointing directions). This contamination is also taken into account in the likelihood ( $\mathcal{L}$, see Section 4) and is estimated to be of $\sim 10 \%(\sim 40 \%)$ of the signal integrated in $\mathrm{ON}$ for pointing mode $\mathcal{A}(\mathcal{B})$, a factor that further affects the sensitivity. 


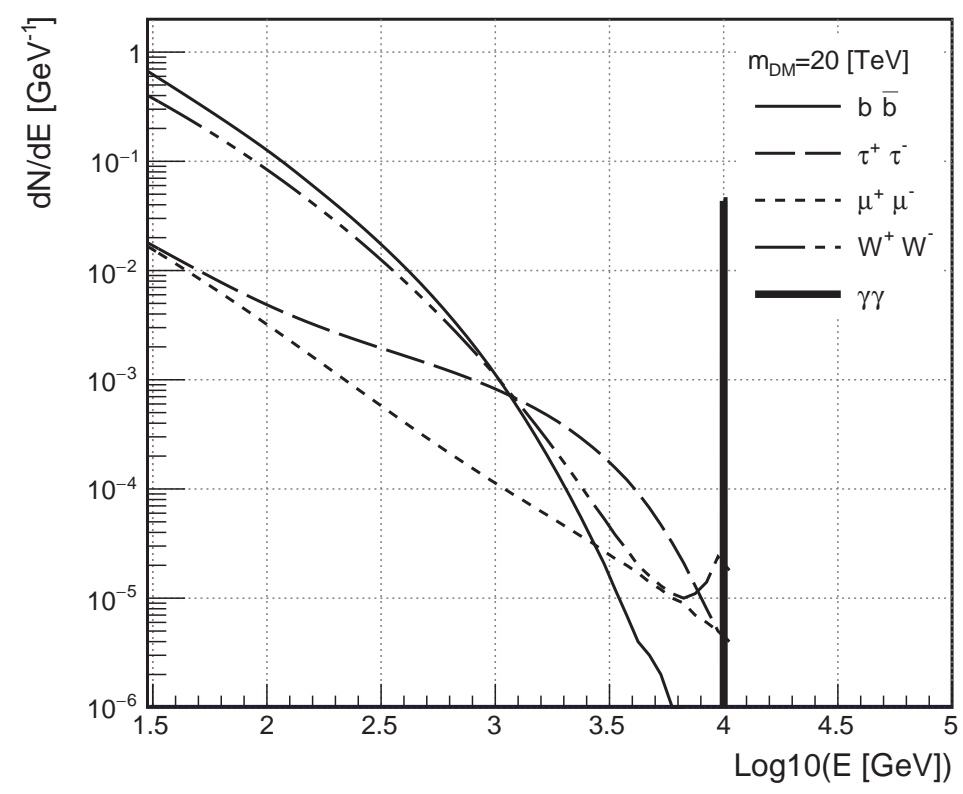

Figure 2: Average gamma-ray spectra $(d N / d E)$ as a function of the gamma-ray energy $(E)$ due to prompt emission for the decay channels $b \bar{b}, \mu^{+} \mu^{-}, \tau^{+} \tau^{-}, W^{+} W^{-}$and $\gamma \gamma$ for a $20 \mathrm{TeV}$ DM particle.

\subsection{Gamma-ray shower discrimination and final event selection}

As seen in Equation 1 and 2, the expected gamma-ray flux depends on the instrument-related parameters $\theta_{\min }$ and $\theta_{\max }$ and also depends on the cut on hadronness $h_{\mathrm{c}}$, optimized independently in each energy bin. Both cuts are optimized based on their expected sensitivity to the DM decay lifetime (as will be introduced in Section 4). The optimal selection values are then $h_{\mathrm{c}}$, selected for a $\mathrm{MC}$ event efficiency of $80 \%, \theta_{\min }=$ $0.1^{\circ}$ and $\theta_{\max }=0.33^{\circ}$ (note that $\theta_{\max }$ is already close to $0.4^{\circ}$, the wobble distance at which data were taken). The effective $J$-factor for those cut 11 is $\sim 0.99 \times 10^{18} \mathrm{GeV} \mathrm{cm}^{-2}$.

\section{Dark matter decay search}

Following Ahnen et al. (2016b), we use the PYTHIA simulation package version 8.205 (Sjöstrand et al., 2015) to compute the average gamma-ray spectrum per decay process $(\mathrm{d} N / \mathrm{d} E)$ for DM particles of masses between $200 \mathrm{GeV}$ and $200 \mathrm{TeV}$ decaying into the SM pairs $b \bar{b}, \tau^{+} \tau^{-}, \mu^{+} \mu^{-}, W^{+} W^{-}$and $\gamma \gamma$. For each channel and mass, we average the gamma-ray spectrum resulting from $10^{7}$ decay events of a generic resonance with mass $m_{D M}$ into the considered pair (see Figure 2). For each simulated event, we trace all the decay chains, including the muon radiative decay $\left(\mu^{-} \rightarrow e^{-} \bar{v}_{e} v_{\mu} \gamma\right.$, not active in PYTHIA by default), down to stable particles. To search for DM in the Perseus CG, we use a binned likelihood method developed for indirect DM searches with IACTs (Aleksić et al., 2012a).

\footnotetext{
${ }^{1}$ Estimated by $J_{\mathrm{dec}} \cdot N^{\theta_{\min }<\theta<\theta_{\max }} / N^{\theta<1.5^{\circ}}$, with $N^{\theta<1.5^{\circ}}$ and $N^{\theta_{\min }<\theta<\theta_{\max }}$ the number of simulated gamma-ray events, following the spatial distribution expected for the decay DM signal from Perseus CG, detected before and after the angular cuts, respectively.
} 
The binned likelihood used in our analysis is written as

$$
\begin{aligned}
& \mathcal{L}\left(1 / \tau_{\mathrm{DM}} ; \boldsymbol{v} \mid \mathcal{D}\right) \\
= & \prod_{i=1}^{N_{\text {samples }}} \mathcal{K}\left(\kappa_{i} \mid \kappa_{\mathrm{Obs}, i}, \sigma_{\kappa, i}\right) \\
& \times \prod_{j=1}^{N_{\text {bins }}}\left[\frac{\left(g_{i j}\left(\tau_{\mathrm{DM}}\right)+b_{i j}+f_{i j}\right)^{N_{\mathrm{ON}, i j}}}{N_{\mathrm{ON}, i j} !} e^{-\left(g_{i j}\left(\tau_{\mathrm{DM}}\right)+b_{i j}+f_{i j}\right)}\right. \\
& \left.\times \frac{\left(\kappa_{i} b_{i j}+g_{i j}^{\mathrm{OFF}}\left(\tau_{\mathrm{DM}}\right)\right)^{N_{\mathrm{OFF}, i j}}}{N_{\mathrm{OFF}, i j} !} e^{-\left(\kappa_{i} b_{i j}+g_{i j}^{\mathrm{OFF}}\left(\tau_{\mathrm{DM}}\right)\right)}\right],
\end{aligned}
$$

where $v$ collectively refers to the nuisance parameters and $\mathcal{D}$ to the data being

$$
\begin{aligned}
& \boldsymbol{v}=\left\{b_{i j}\right\}, \kappa_{i} \\
& \mathcal{D}=\left(N_{\mathrm{ON}, i j}, N_{\mathrm{OFF}, i j}\right) .
\end{aligned}
$$

The index $i$ refers to the independent datasets (described in Table 2), and $j$ to the bins of estimated energy. The parameters $g_{i j}$ and $g_{i j}^{\mathrm{OFF}}$ are the estimated number of DM signal events for the ON and OFF regions, respectively; the parameters $b_{i j}$ are the estimated number of background events; $N_{\mathrm{ON}, i j}$ are the number of observed events in the $\mathrm{ON}$ region and $N_{\mathrm{OFF}, i j}$ is the number of observed events in the corresponding OFF bin; $\mathcal{K}$ is the likelihood function for $\kappa_{i}$ (the $\mathrm{OFF} / \mathrm{ON}$ acceptance ratio), parametrized by a Gaussian function with mean $\kappa_{\mathrm{obs}, i}$ and variance $\sigma_{\kappa, i}^{2}$, which includes statistical and systematic uncertainties, added in quadrature assuming Poisson statistics. We consider a systematic uncertainty for the parameter $\kappa_{i}, \sigma_{\kappa}^{\text {sys }}=0.015 \kappa_{\mathrm{obs}, i}$, a value that has been established in (Aleksić et al. 2016a). $f_{i j}$ (considered as fixed parameters in this analysis, in order not to lose the convergence of the likelihood) are the estimated number of foreground events from NGC 1275 (computed by extrapolating from the estimated number of gamma-ray excess-events within $\mathcal{R} 1$ around NGC 1275, see Figure 1). We infer the number of expected events reconstructed inside $\mathcal{R} 2$ taking into account the instrument's angular point spread function computed from a point-like MC representative for each analyzed data sample (same hardware stable period, same zenith range, and weighted to reproduce NGC1275's spectra measured in Ahnen et al. 2016a). $b_{i j}$ and $\kappa_{i}$ are nuisance parameters, whereas the estimated number of signal events $g_{i j}$ and $g_{i j}^{\text {OFF }}$ depend on the free parameter $\tau_{\mathrm{DM}}$ through

$$
\begin{aligned}
g_{i j}\left(\tau_{\mathrm{DM}}\right) & =T_{\mathrm{obs}, i} \int_{E_{\text {min }, j}^{\prime}}^{E_{\text {max }, j}^{\prime}} \mathrm{d} E^{\prime} \int_{0}^{\infty} \mathrm{d} E \frac{\mathrm{d} \phi\left(\tau_{\mathrm{DM}}\right)}{\mathrm{d} E} A_{\mathrm{eff}}(E)_{i} G\left(E^{\prime} \mid E\right)_{i}, \\
g_{i j}^{\mathrm{OFF}}\left(\tau_{\mathrm{DM}}\right) & =T_{\mathrm{obs}, i} \int_{E_{\text {min }, j}^{\prime}}^{E_{\text {max }, j}} \mathrm{~d} E^{\prime} \int_{0}^{\infty} \mathrm{d} E \frac{\mathrm{d} \phi\left(\tau_{\mathrm{DM}}\right)}{\mathrm{d} E} A_{\mathrm{eff}}(E)_{i} \epsilon(E)_{i} G\left(E^{\prime} \mid E\right)_{i} .
\end{aligned}
$$

$T_{\mathrm{obs}, i}$ is the total observation time, $E$ and $E^{\prime}$ the true and estimated gamma-ray energy respectively, and $E_{\min , j}^{\prime}$ and $E_{\max , j}^{\prime}$ the minimum and maximum energies of the $j$-th energy bin. Finally, $G$ is the probability density function for the energy estimator $E^{\prime}$ for true energy $E, A_{\mathrm{eff}}$ is the effective collection area for $\mathcal{R} 2$ angular cuts computed from the tailored MC sample introduced in Section 3 (that takes into account the expected morphology of the gamma-ray signal and the instrument angular resolution), and $\epsilon_{i}$, the ratio between expected number of signal events in the OFF and ON regions, obtained from the same MC sample. 


\begin{tabular}{l|c}
$\mathcal{A}$ \\
\hline \hline Hardware stable period & $\mathcal{P} 1, \mathcal{P} 2, \mathcal{P} 3, \mathcal{P} 4, \mathcal{P} 5, \mathcal{P} 6$ \\
\hline Zenith angle & {$[5-35]$} \\
\hline Wobble pointing & $W 0.4\left(+058^{\circ},+157^{\circ},+238^{\circ},+337^{\circ}\right)$ \\
\hline \hline
\end{tabular}

\begin{tabular}{l|c}
$\mathcal{B}$ \\
\hline \hline Hardware stable period & $\mathcal{P} 2, \mathcal{P} 3, \mathcal{P} 4, \mathcal{P} 5, \mathcal{P} 6$ \\
\hline Zenith angle & {$[5-35],[35-50]$} \\
\hline Wobble pointing & $W 0.26\left(+108^{\circ},+288^{\circ}\right)$ \\
\hline \hline
\end{tabular}

Table 2: List of the different bins for which independent IRFs (and hence, independent likelihoods) are defined.

We define the profile likelihood ratio as

$$
\lambda_{P}\left(1 / \tau_{\mathrm{DM}} \mid \mathcal{D}\right)=\frac{\mathcal{L}\left(1 / \tau_{\mathrm{DM}} ; \hat{\hat{v}} \mid \mathcal{D}\right)}{\mathcal{L}\left(\widehat{1 / \tau_{\mathrm{DM}}} ; \hat{\boldsymbol{v}} \mid \mathcal{D}\right)}
$$

where $\widehat{1 / \tau_{\mathrm{DM}}}$ and $\hat{v}$ are the values maximizing $\mathcal{L}\left(\mathcal{L}\right.$ is linear in $\left.1 / \tau_{\mathrm{DM}}\right)$, and $\hat{v}$ the value that maximizes $\mathcal{L}$ for a fixed $1 / \tau_{\mathrm{DM}}$ (when performing the maximization we restricted the value of the lifetime to the physical range, $\left.1 / \tau_{\mathrm{DM}} \geq 0\right)$. Lower limits in $\tau_{\mathrm{DM}}$ at $95 \% \mathrm{CL}\left(\tau_{\mathrm{DM}}^{\mathrm{LL}}\right)$ are given for

$$
-2 \ln \lambda_{P}\left(1 / \tau_{\mathrm{DM}}^{\mathrm{LL}} \mid \mathcal{D}\right)=2.71
$$

We approximate the expected sensitivity to the DM decay lifetime $\tau_{\mathrm{DM}}^{\mathrm{svt}}$ as

$$
\tau_{\mathrm{DM}}^{\mathrm{svt}}=\left(1 / \tau_{\mathrm{DM}}^{\mathrm{LL}}-\widehat{1 / \tau_{\mathrm{DM}}}\right)^{-1} .
$$

The null hypothesis is the case with no DM signal $\left(1 / \tau_{\mathrm{DM}}=0\right)$, while the test hypotheses are built considering the flux computed using Equation 2, under the assumption of different DM particles with masses from $200 \mathrm{GeV}$ to $200 \mathrm{TeV}$ for pure SM decays. The dataset is divided into $N_{\text {samples }}=42$ independent subset ${ }^{2}$ according to the two observational pointing schemes $(\mathcal{A}$ and $\mathcal{B})$, the wobble pointing positions, the different hardware stable periods, and two zenith ranges $\left(15-35^{\circ}\right.$ and $\left.35-50^{\circ}\right)$. Each likelihood term is linked to the rest of the terms through the common physical parameter $\tau_{\mathrm{DM}}$.

\section{Results and Discussion}

We performed a search for decaying DM in the Perseus CG using $202 \mathrm{~h}$ of data passing a thorough selection as described in Section 3, for DM particles decaying into $b \bar{b}, \tau^{+} \tau^{-}, \mu^{+} \mu^{-}, W^{+} W^{-}$, and $\gamma \gamma$, with masses between $200 \mathrm{GeV}$ and $200 \mathrm{TeV}$ (for decays into $\gamma \gamma$, the scanned mass range has been reduced between $200 \mathrm{GeV}$ and $20 \mathrm{TeV}$, since for such spectra for larger masses the number of expected gamma-ray events

\footnotetext{
${ }^{2}$ Four subsamples, out of the naively expected 44 from Table 2 were excluded from the search since almost no data survived the cuts introduced in Table 1. Moreover, two extra pointings (included in Table 1 but not described in Table 2) were taken at the beginning of the campaign at the same wobble distance but with different orientation, before optimizing pointing mode $\mathcal{A}$ and $\mathcal{B}$. These two subsamples (accounting each of them for $\sim 17 \mathrm{~h}$ ) are also included in the analysis.
} 

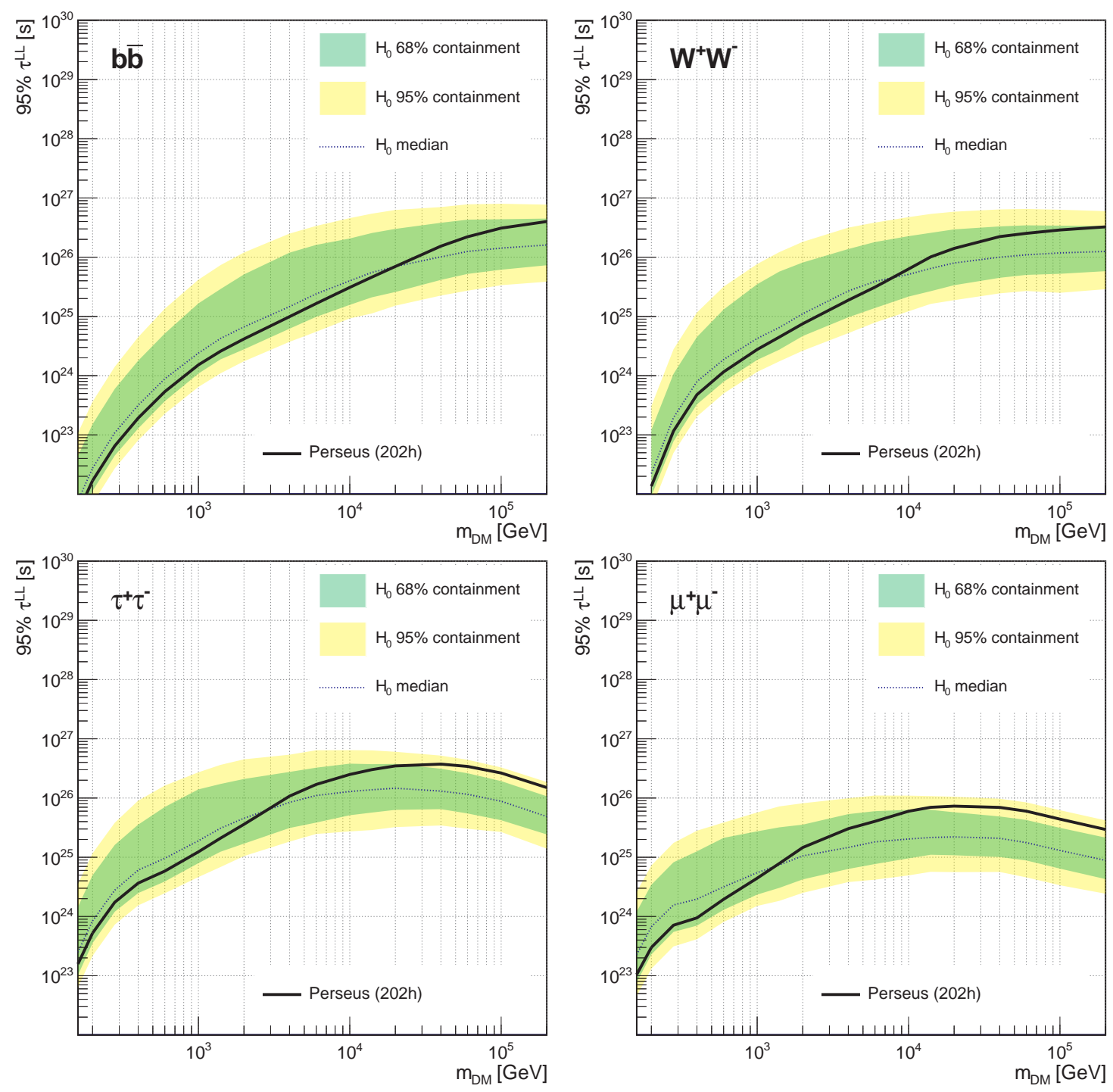

Figure 3: $95 \%$ CL lower limit on the DM decay lifetime (solid line) in the $b \bar{b}$ (top-left), $W^{+} W^{-}$(top-right), $\tau^{+} \tau^{-}$(bottom-left) and $\mu^{+} \mu^{-}$(bottom-right) channels using $202 \mathrm{~h}$ of Perseus CG data. The expected limit (dashed line) and the two sided $68 \%$ and $95 \%$ containment bands are also shown.

detected by MAGIC in the observation time is lower than 1). 95\% CL lower limits on the DM particle decay lifetime $\tau_{\mathrm{DM}}$ for each decay channel are obtained with a binned likelihood analysis ( $80 \mathrm{GeV}$ to $10 \mathrm{TeV}$ in 10 logarithmic-spaced bin $s^{3}$ ) using $J_{\mathrm{dec}}=1.5 \times 10^{19} \mathrm{GeV} \mathrm{cm}^{-2}$ (see Section 2). The results for leptonic and hadronic decays are shown in Figure 3 where also reported are the two-sided 68\% and 95\% containment bands and the median for the null hypothesis, computed from the distribution of the lower limits obtained from the analysis of 300 realizations of the null hypothesis. This consist of MC simulations in which both $\mathrm{ON}$ and OFF regions are generated from pure background probability density functions, assuming both similar exposures for the real data, and $\kappa_{i}$ taken as a nuisance parameter in the likelihood function. We reach sensitivities $\tau_{\mathrm{DM}}>10^{26} \mathrm{~s}$ where no evidence for decaying DM is found in either decay mode.

\footnotetext{
${ }^{3}$ Empty bins are merged with neighbouring ones.
} 

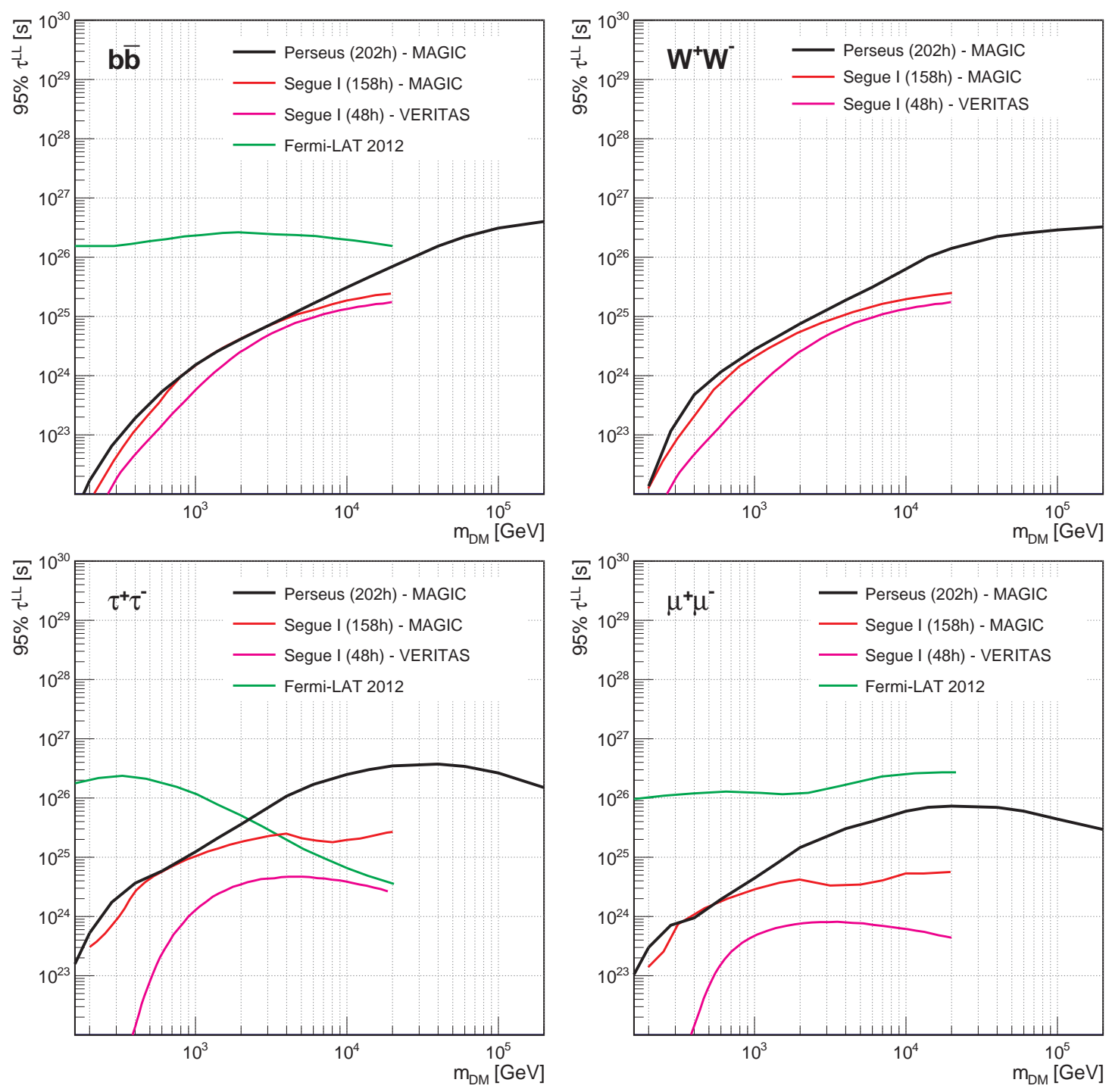

Figure 4: Comparison of the 95\% CL lower limit on the DM decay lifetime from the Perseus CG (solid black line) with similar measurements in the dSph Segue I by the MAGIC (Aleksić et al. 2014c red line) and VERITAS (Aliu et al., 2012, pink line) collaborations. The limits obtained from the diffuse galactic center from the Fermi-LAT collaboration (Ackermann et al. 2012b. green-line) are also shown (limits for $W^{+} W^{-}$not available).

Figure 4 show the comparison of MAGIC lower limits with results from other searches. Decaying DM scenarios are currently investigated with several classes of instruments and for different mass ranges. In the GeV-TeV mass range, the majority of limits make use of the Fermi-LAT instrument, sensitive in the $\mathrm{MeV}-\mathrm{GeV}$ range, in two ways: either combining results from observations of CGs (Dugger et al., 2010, Ke et al., 2011; Zimmer et al., 2011; Huang et al., 2012; Charles et al., 2016; Ackermann et al., 2015a) or making use of the integrated cosmological decaying DM contribution to the extragalactic diffuse light (see, e.g., Cirelli et al., 2012; Cohen et al., 2017), composed of prompt and secondary emission. It must be underlined that these results were not independently validated by the Fermi-LAT collaboration and that, in some cases these limits depend on the model-dependent secondary components. In the TeV energy range, where searches for diffuse emission are hindered because of the limited FoV of ground based IACTs, the 


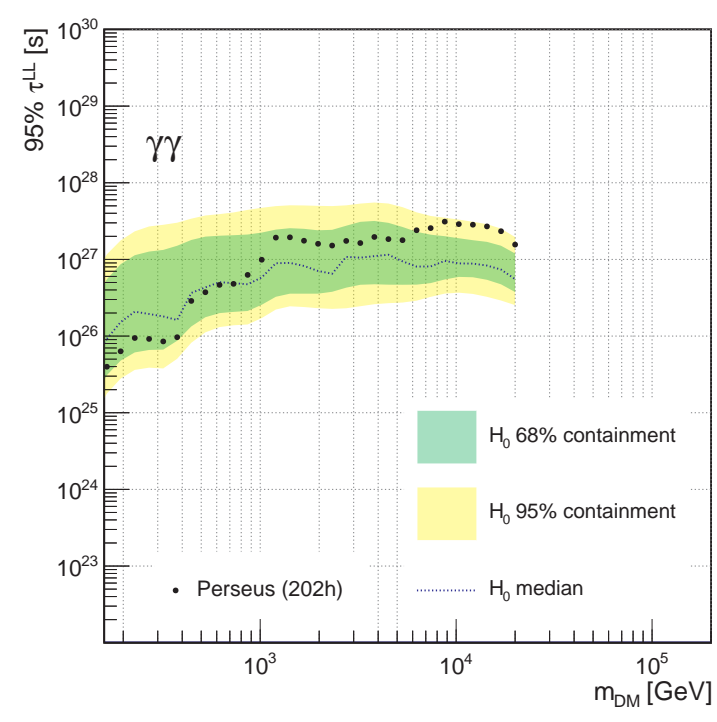

(a)

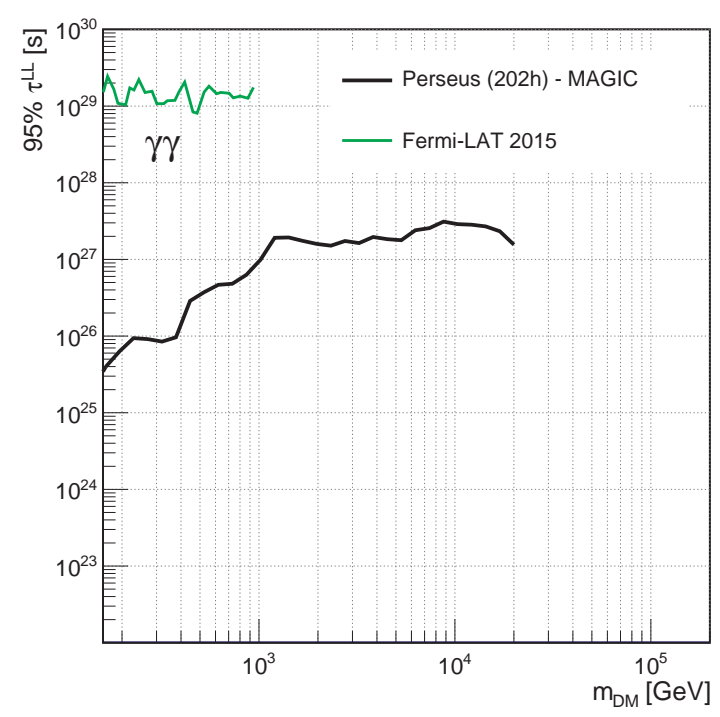

(b)

Figure 5: (left) Same as Figure 3 for DM particles decaying into $\gamma \gamma$. The scanned mass range goes between $200 \mathrm{GeV}$ and $20 \mathrm{TeV}$, since for larger masses the number of expected gamma-ray events detected by MAGIC in the observation time is lower than 1. (right) Same as Figure 4DM particles decaying into $\gamma \gamma$. Fermi-LAT data based on (Ackermann et al. 2015b).

decaying DM case was discussed by Cirelli et al. (2012) showing lower limits on the DM decay lifetime with H.E.S.S. data for the Fornax CG, but again an independent validation from the H.E.S.S. collaboration has not yet been published. At higher energies, the most stringent constraints on certain channels can be obtained with neutrinos with IceCube (Cohen et al., 2017) or ultra-high-energy cosmic rays with the Pierre Auger Observatory, KASKADE, and CASA-MIA (Aab et al., 2015; Kang et al., 2015; Chantell et al., 1997). In the VHE gamma-ray range, our results are compared with previous limits obtained with MAGIC using $158 \mathrm{~h}$ of the dSph Segue 1 (Aleksić et al., 2014c). We also show limits from $48 \mathrm{~h}$ observation of Segue 1 with VERITAS (Aliu et al., 2012) and with Fermi-LAT data on the Galactic Center (Ackermann et al., 2012b). The comparison of these results may suffer from the fact that nuisance parameters are treated differently in different analyses. Fermi-LAT results are more constraining in the low WIMP mass range, up to few hundreds of $\mathrm{GeV}$, depending on the decay channel. However, they are based on significant assumptions on the nature of the diffuse galactic gamma-ray emission, as discussed in Ackermann et al. (2012b). The Perseus results are more constraining than previous dSph limits achieved above few hundreds $\mathrm{GeV}$ and extend previous MAGIC results towards larger WIMP DM masses so far unexplored. From a mere comparison of the astrophysical factors (60 times larger for Perseus than for Segue 1) one would have expected a similarly stronger constraint. However, several factors degrade the sensitivity in this analysis. Primarily, the region of interest is more complex: the presence of known astrophysical emitters in the FoV requires a reduction of the search signal region, and the extension of the DM profile induces a leakage of signal into the OFF region. Secondly, this analysis is made more accurate by additionally taking into account the uncertainty in the background rate estimation, and the different computation of the IRFs, which consider the morphology of the CG emission.

We did not consider the effect of a second gamma-ray contribution coming from the interaction of charged particles (most notably light leptons, generated during the decay process normally after hadronization, fragmentation and decay of prompt products) interacting with the intra-cluster magnetic field (ICMF) of Perseus 
or the cosmic microwave background (CMB). These charged particles may diffuse away from the DM halo although they are expected to be contained by efficient energy loss such as with synchrotron and Inverse Compton (IC) processes. This was investigated for the annihilating DM case in Gómez-Vargas et al. (2013) and Ackermann et al. (2015a), where the authors showed that for DM masses above $50 \mathrm{GeV}$, the contribution from secondary gamma rays can boost the signal up to a factor of 5 for muons and a factor of 2 for taus (Figure 5 of Gómez-Vargas et al., 2013) for the Galactic Center region. Such a contribution would be proportional to the $J$-factor, and therefore be present also in our case. The predominance of the former or the latter, discussed also in Gómez-Vargas et al. (2013), is governed by the intensity of the ICMF. For values larger than $3 \mu \mathrm{G}$, the magnetic field energy density is comparable to that of the CMB photons, that are the seeds for IC up-scatterings. Considering that the Perseus core is expected to have a magnetic field

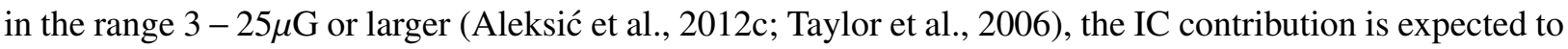
be less relevant due to important synchrotron losses. Above several hundreds $\mathrm{GeV}$, our results are therefore conservative and could be only slightly more stringent in case secondary emission is considered.

Finally, the results for monochromatic line decays are shown in Figure 5a where we reach sensitivities $\tau_{\mathrm{DM}}>10^{27} \mathrm{~s}$. Again, no evidence for decaying DM is found. In Figure $5 \mathrm{~b}$, Perseus results for line-like spectra are put into context and compared with the Fermi-LAT data of Ackermann et al. $(2015 \mathrm{~b})$. The Fermi-LAT collaboration has published several studies on spectral line searches (Abdo et al., 2010; Ackermann et al., 2012a, 2013). In their latest work (Ackermann et al., 2015b), Fermi-LAT updated their results using 5.8 years of Pass 8 data and an optimized region of interest according to different DM realizations. One can see that Fermi-LAT data are more constraining below the TeV mass scale while Perseus results are the most constraining results at the low $\mathrm{TeV}$ mass range and extend the current scanned mass range of decaying WIMPs to larger values.

\section{Summary and Conclusions}

Clusters of galaxies are optimal targets for decaying DM searches, given their huge expected DM content. The MAGIC telescopes have observed the Perseus CG for about $400 \mathrm{~h}$ over several years. The data sample was used in this paper to search for decaying DM in the Perseus CG core. The analysis is made more complex by the presence of the central radio galaxy NGC 1275, a known bright gamma-ray emitter with variable flux, as well as by the more peripheral radio galaxy IC 310. Furthermore, the putative signal region extends outwards to a radius a few times the telescope angular resolution. This necessitated the development of a tailored MC sample and the inclusion of extra terms in the likelihood function with respect to a standard analysis. Out of the full data sample, $202 \mathrm{~h}$ have been selected for our study. No evidence of a DM signal has been found. From this result, we cast lower limits on the decay lifetime $\tau_{\mathrm{DM}}$ of WIMP DM with masses between $200 \mathrm{GeV}$ and $200 \mathrm{TeV}$ for several "pure" (i.e. 100\% branching ratio) decay channels $b \bar{b}, \tau^{+} \tau^{-}, W^{+} W^{-}, \mu^{+} \mu^{-}$as well as for "pure" $\gamma \gamma$ decay line. We have reached strong limits on the order of $\tau_{\mathrm{DM}}=2 \times 10^{26} \mathrm{~s}$ for a $10 \mathrm{TeV} \mathrm{DM}$ particle decaying into $\tau^{+} \tau^{-}$and $\tau_{\mathrm{DM}}=3 \times 10^{27} \mathrm{~s}$ for a $10 \mathrm{TeV} \mathrm{DM}$ particle decaying into $\gamma \gamma$. Our limits improve previous MAGIC results and are the most constraining limits on the decay lifetime of DM particles based on observations from ground-based gamma-ray instruments.

It is unlikely that dwarf satellite galaxies can provide stronger constraints on decaying DM scenarios than the ones provided by CGs. Improving current limits on decaying DM particles with this technique would

\footnotetext{
${ }^{4}$ Using the lower limit on decay lifetime computed in the region of interest dubbed R180 in their paper, optimized to search for spectral lines from DM decay
} 
require a significant increase of observation time on CGs, which is unlikely in the close future with the current generation of IACT. Therefore, these results are unlikely to be further improved until the next generation of Cherenkov telescopes, like the Cherenkov Telescope Array (CTA, Acharya et al., 2017), becomes active.

Acknowledgements. We would like to thank the Instituto de Astrofísica de Canarias for the excellent working conditions at the Observatorio del Roque de los Muchachos in La Palma. The financial support of the German BMBF and MPG, the Italian INFN and INAF, the Swiss National Fund SNF, the ERDF under the Spanish MINECO (FPA2015-69818-P, FPA2012-36668, FPA2015-68378-P, FPA2015-69210C6-2-R, FPA2015-69210-C6-4-R, FPA2015-69210-C6-6-R, AYA2015-71042-P, AYA2016-76012-C3-1-P, ESP2015-71662-C2-2-P, CSD2009-00064), and the Japanese JSPS and MEXT is gratefully acknowledged. This work was also supported by the Spanish Centro de Excelencia "Severo Ochoa" SEV-2012-0234 and SEV-2015-0548, and Unidad de Excelencia "María de Maeztu" MDM-2014-0369, by the Croatian Science Foundation (HrZZ) Project IP-2016-06-9782 and the University of Rijeka Project 13.12.1.3.02, by the DFG Collaborative Research Centers SFB823/C4 and SFB876/C3, the Polish National Research Centre grant UMO-2016/22/M/ST9/00382 and by the Brazilian MCTIC, CNPq and FAPERJ. The work of the author M. Vazquez Acosta is financed with grant RYC-2013-14660 of MINECO.

We are also indepted to G. Brunetti, C. Combet, D. Maurin, S. Zimmer for valid inputs and specially M.A. Sanchez Conde and F. Zandanel, for fruitful discussions and insights.

\section{References}

A. Aab et al. The Pierre Auger Observatory: Contributions to the 34th International Cosmic Ray Conference (ICRC 2015). 2015. URL https://inspirehep.net/record/1393211/files/arXiv:1509.03732.pdf

A. A. Abdo et al. Measurement of the Cosmic Ray e+ plus e- spectrum from $20 \mathrm{GeV}$ to $1 \mathrm{TeV}$ with the Fermi Large Area Telescope. Phys. Rev. Lett., 102:181101, 2009. doi: 10.1103/PhysRevLett.102.181101.

A. A. Abdo et al. Fermi LAT Search for Photon Lines from 30 to $200 \mathrm{GeV}$ and Dark Matter Implications. Phys. Rev. Lett., 104: 091302, 2010. doi: 10.1103/PhysRevLett.104.091302.

B. S. Acharya et al. Science with the Cherenkov Telescope Array. 2017.

M. Ackermann et al. Fermi LAT observations of cosmic-ray electrons from $7 \mathrm{GeV}$ to $1 \mathrm{TeV}$. Phys. Rev., D82:092004, 2010. doi: 10.1103/PhysRevD.82.092004.

M. Ackermann et al. Fermi LAT Search for Dark Matter in Gamma-ray Lines and the Inclusive Photon Spectrum. Phys. Rev., D86: 022002, 2012a. doi: 10.1103/PhysRevD.86.022002.

M. Ackermann et al. Constraints on the Galactic Halo Dark Matter from Fermi-LAT Diffuse Measurements. Astrophys. J., 761: 91, 2012b. doi: 10.1088/0004-637X/761/2/91.

M. Ackermann et al. Search for Gamma-ray Spectral Lines with the Fermi Large Area Telescope and Dark Matter Implications. Phys. Rev., D88:082002, 2013. doi: 10.1103/PhysRevD.88.082002.

M. Ackermann et al. Search for extended gamma-ray emission from the Virgo galaxy cluster with Fermi-LAT. Astrophys. J., 812 (2):159, 2015a. doi: 10.1088/0004-637X/812/2/159.

M. Ackermann et al. Updated search for spectral lines from Galactic dark matter interactions with pass 8 data from the Fermi Large Area Telescope. Phys. Rev., D91(12):122002, 2015b. doi: 10.1103/PhysRevD.91.122002.

O. Adriani et al. An anomalous positron abundance in cosmic rays with energies 1.5-100 GeV. Nature, 458:607-609, 2009. doi: 10.1038/nature07942.

M. Aguilar et al. First Result from the Alpha Magnetic Spectrometer on the International Space Station: Precision Measurement of the Positron Fraction in Primary Cosmic Rays of 0.5350 GeV. Phys. Rev. Lett., 110:141102, 2013. doi: 10.1103/PhysRevLett. 110.141102

M. L. Ahnen et al. Deep observation of the NGC1275 region with MAGIC: search of diffuse $\gamma$-ray emission from cosmic rays in the Perseus cluster. Astron. Astrophys., 589:A33, 2016a. doi: 10.1051/0004-6361/201527846.

M. L. Ahnen et al. Limits to dark matter annihilation cross-section from a combined analysis of MAGIC and Fermi-LAT observations of dwarf satellite galaxies. JCAP, 1602(02):039, 2016b. doi: 10.1088/1475-7516/2016/02/039. 
M. L. Ahnen et al. Performance of the MAGIC telescopes under moonlight. Astropart. Phys., 94:29-41, 2017. doi: 10.1016/j. astropartphys.2017.08.001.

M. L. Ahnen et al. Indirect dark matter searches in the dwarf satellite galaxy Ursa Major II with the MAGIC Telescopes. JCAP, 1803(03):009, 2018. doi: 10.1088/1475-7516/2018/03/009.

J. Albert et al. Implementation of the Random Forest Method for the Imaging Atmospheric Cherenkov Telescope MAGIC. Nucl. Instrum. Meth., A588:424-432, 2008. doi: 10.1016/j.nima.2007.11.068.

J. Aleksić, J. Rico, and M. Martinez. Optimized analysis method for indirect dark matter searches with Imaging Air Cherenkov Telescopes. JCAP, 1210:032, 2012a. doi: 10.1088/1475-7516/2012/10/032.

J. Aleksić et al. MAGIC Gamma-Ray Telescope Observation of the Perseus Cluster of Galaxies: Implications for Cosmic Rays, Dark Matter and NGC 1275. Astrophys. J., 710:634-647, 2010a. doi: 10.1088/0004-637X/710/1/634.

J. Aleksić et al. Detection of very high energy gamma-ray emission from the Perseus cluster head-tail galaxy IC 310 by the MAGIC telescopes. Astrophys. J., 723:L207, 2010b. doi: 10.1088/2041-8205/723/2/L207.

J. Aleksić et al. Performance of the MAGIC stereo system obtained with Crab Nebula data. Astropart. Phys., 35:435-448, 2012b. doi: 10.1016/j.astropartphys.2011.11.007.

J. Aleksić et al. Constraining Cosmic Rays and Magnetic Fields in the Perseus Galaxy Cluster with TeV observations by the MAGIC telescopes. Astron. Astrophys., 541:A99, 2012c. doi: 10.1051/0004-6361/201118502.

J. Aleksić et al. Detection of very high energy gamma-ray emission from NGC 1275 by the MAGIC telescopes. Astron. Astrophys., 539:L2, 2012d. doi: 10.1051/0004-6361/201118668.

J. Aleksić et al. Rapid and multiband variability of the TeV bright active nucleus of the galaxy IC 310. Astron. Astrophys., 563: A91, 2014a. doi: 10.1051/0004-6361/201321938.

J. Aleksić et al. Contemporaneous observations of the radio galaxy NGC 1275 from radio to very high energy $\gamma$-rays. Astron. Astrophys., 564:A5, 2014b. doi: 10.1051/0004-6361/201322951.

J. Aleksić et al. Optimized dark matter searches in deep observations of Segue 1 with MAGIC. JCAP, 1402:008, 2014c. doi: 10.1088/1475-7516/2014/02/008.

J. Aleksić et al. Black hole lightning due to particle acceleration at subhorizon scales. Science, 346:1080-1084, 2014d. doi: $10.1126 /$ science. 1256183 .

J. Aleksić et al. The major upgrade of the MAGIC telescopes, Part II: A performance study using observations of the Crab Nebula. Astropart. Phys., 72:76-94, 2016a. doi: 10.1016/j.astropartphys.2015.02.005.

J. Aleksić et al. The major upgrade of the MAGIC telescopes, Part I: The hardware improvements and the commissioning of the system. Astropart. Phys., 72:61-75, 2016b. doi: 10.1016/j.astropartphys.2015.04.004.

E. Aliu et al. VERITAS Deep Observations of the Dwarf Spheroidal Galaxy Segue 1. Phys. Rev., D85:062001, 2012. doi: 10.1103/PhysRevD.85.062001,10.1103/PhysRevD.91.129903. [Erratum: Phys. Rev.D91,no.12,129903(2015)].

S. Ando and K. Ishiwata. Constraints on decaying dark matter from the extragalactic gamma-ray background. JCAP, 1505(05): 024, 2015. doi: 10.1088/1475-7516/2015/05/024.

S. Ando and A. Kusenko. Interactions of keV sterile neutrinos with matter. Phys. Rev., D81:113006, 2010. doi: 10.1103/PhysRevD. 81.113006.

V. Berezinsky, A. Masiero, and J. W. F. Valle. Cosmological signatures of supersymmetry with spontaneously broken R-parity. Phys. Lett., B266:382-388, 1991. doi: 10.1016/0370-2693(91)91055-Z.

L. Bergstrom, P. Ullio, and J. H. Buckley. Observability of gamma-rays from dark matter neutralino annihilations in the Milky Way halo. Astropart. Phys., 9:137-162, 1998. doi: 10.1016/S0927-6505(98)00015-2.

C. Boehm, T. A. Ensslin, and J. Silk. Can Annihilating dark matter be lighter than a few GeVs? J. Phys., G30:279-286, 2004. doi: 10.1088/0954-3899/30/3/004.

O. Catá, A. Ibarra, and S. Ingenhtt. Dark matter decay through gravity portals. Phys. Rev., D95(3):035011, 2017. doi: 10.1103/ PhysRevD.95.035011.

M. C. Chantell et al. Limits on the isotropic diffuse flux of ultrahigh-energy gamma radiation. Phys. Rev. Lett., 79:1805-1808, 1997. doi: 10.1103/PhysRevLett.79.1805.

E. Charles et al. Sensitivity Projections for Dark Matter Searches with the Fermi Large Area Telescope. Phys. Rept., 636:1-46, 2016. doi: 10.1016/j.physrep.2016.05.001.

X.-L. Chen and M. Kamionkowski. Particle decays during the cosmic dark ages. Phys. Rev., D70:043502, 2004. doi: 10.1103/ PhysRevD.70.043502.

Y. Chen, T. H. Reiprich, H. Bohringer, Y. Ikebe, and Y. Y. Zhang. Statistics of X-ray observables for the cooling-core and noncooling core galaxy clusters. Astron. Astrophys., 2007. doi: 10.1051/0004-6361:20066471. [Astron. Astrophys.466,805(2007)].

M. Cirelli, E. Moulin, P. Panci, P. D. Serpico, and A. Viana. Gamma ray constraints on Decaying Dark Matter. Phys. Rev., D86: 083506, 2012. doi: 10.1103/PhysRevD.86.083506,10.1103/PhysRevD.86.109901.

T. Cohen, K. Murase, N. L. Rodd, B. R. Safdi, and Y. Soreq. $\gamma$-ray Constraints on Decaying Dark Matter and Implications for IceCube. Phys. Rev. Lett., 119(2):021102, 2017. doi: 10.1103/PhysRevLett.119.021102. 
L. Dugger, T. E. Jeltema, and S. Profumo. Constraints on Decaying Dark Matter from Fermi Observations of Nearby Galaxies and Clusters. JCAP, 1012:015, 2010. doi: 10.1088/1475-7516/2010/12/015.

J. L. Feng. Dark Matter Candidates from Particle Physics and Methods of Detection. Ann. Rev. Astron. Astrophys., 48:495-545, 2010. doi: 10.1146/annurev-astro-082708-101659.

L. Feng, R.-Z. Yang, H.-N. He, T.-K. Dong, Y.-Z. Fan, and J. Chang. AMS-02 positron excess: new bounds on dark matter models and hint for primary electron spectrum hardening. Phys. Lett., B728:250-255, 2014. doi: 10.1016/j.physletb.2013.12.012.

V. P. Fomin, A. A. Stepanian, R. C. Lamb, D. A. Lewis, M. Punch, and T. C. Weekes. New methods of atmospheric Cherenkov imaging for gamma-ray astronomy. 1: The False source method. Astropart. Phys., 2:137-150, 1994. doi: 10.1016/0927-6505(94)90036-1.

K. Freese. Review of Observational Evidence for Dark Matter in the Universe and in upcoming searches for Dark Stars. EAS Publ. Ser., 36:113-126, 2009. doi: 10.1051/eas/0936016.

C. Fruck, M. Gaug, R. Zanin, D. Dorner, D. Garrido, R. Mirzoyan, and L. Font. A novel LIDAR-based Atmospheric Calibration Method for Improving the Data Analysis of MAGIC. In Proceedings, 33rd International Cosmic Ray Conference (ICRC2013): Rio de Janeiro, Brazil, July 2-9, 2013, page 1054, 2014. URL/http://inspirehep.net/record/1285998/files/arXiv: 1403.3591.pdf

M. Garny, A. Ibarra, D. Tran, and C. Weniger. Gamma-ray lines from radiative dark matter decay. Journal of Cosmology and Astroparticle Physics, 2011(01):032-032, 2011. ISSN 1475-7516. doi: 10.1088/1475-7516/2011/01/032. URL http:// stacks. iop.org/1475-7516/2011/i=01/a=032?key=crossref. a672f146155cad875c8a0c2c07f155c0

G. A. Gómez-Vargas, M. A. Sánchez-Conde, J.-H. Huh, M. Peiró, F. Prada, A. Morselli, A. Klypin, D. G. Cerdeo, Y. Mambrini, and C. Muoz. Constraints on WIMP annihilation for contracted dark matterin the inner Galaxy with the Fermi-LAT. JCAP, 1310:029, 2013. doi: 10.1088/1475-7516/2013/10/029.

K. Griest and M. Kamionkowski. Unitarity Limits on the Mass and Radius of Dark Matter Particles. Phys. Rev. Lett., 64:615, 1990. doi: 10.1103/PhysRevLett.64.615.

L. Hernquist. An Analytical Model for Spherical Galaxies and Bulges. Astrophys. J., 356:359, 1990. doi: 10.1086/168845.

X. Huang, G. Vertongen, and C. Weniger. Probing Dark Matter Decay and Annihilation with Fermi LAT Observations of Nearby Galaxy Clusters. JCAP, 1201:042, 2012. doi: 10.1088/1475-7516/2012/01/042.

A. Ibarra. Gamma-ray lines from dark matter decay. Journal of physics Conference Series, 384:012001, 2012. doi: doi:10.1088/ 1742-6596/384/1/012001. URL http://iopscience.iop.org/article/10.1088/1742-6596/384/1/012001/pdf

T. E. Jeltema, J. Kehayias, and S. Profumo. Gamma Rays from Clusters and Groups of Galaxies: Cosmic Rays versus Dark Matter. Phys. Rev., D80:023005, 2009. doi: 10.1103/PhysRevD.80.023005.

D. Kang et al. A limit on the diffuse gamma-rays measured with KASCADE-Grande. J. Phys. Conf. Ser., 632(1):012013, 2015. doi: 10.1088/1742-6596/632/1/012013.

J. Ke, M. Luo, L. Wang, and G. Zhu. Gamma-rays from Nearby Clusters: Constraints on Selected Decaying Dark Matter Models. Phys. Lett., B698:44-51, 2011. doi: 10.1016/j.physletb.2011.02.055.

T.-P. Li and Y.-Q. Ma. Analysis methods for results in gamma-ray astronomy. Astrophysical Journal, 272:317-324, Sept. 1983. doi: $10.1086 / 161295$.

A. Moliné, M. A. Sánchez-Conde, S. Palomares-Ruiz, and F. Prada. Characterization of subhalo structural properties and implications for dark matter annihilation signals. Mon. Not. Roy. Astron. Soc., 466(4):4974-4990, 2017. doi: 10.1093/mnras/stx026.

J. F. Navarro, C. S. Frenk, and S. D. M. White. The Structure of cold dark matter halos. Astrophys. J., 462:563-575, 1996. doi: $10.1086 / 177173$.

J. Palacio. Indirect dark matter searches on the Triangulum II dwarf spheroidal galaxy and on the Perseus galaxy cluster with the MAGIC Telescopes. PhD thesis, Universitat Autonoma de Barcelona, 2018. URL http://www.tdx.cat/handle/10803/ 462764

P. J. E. Peebles. Principles of physical cosmology. 1994.

A. Pinzke and C. Pfrommer. Simulating the gamma-ray emission from galaxy clusters: a universal cosmic ray spectrum and spatial distribution. Mon. Not. Roy. Astron. Soc., 409:449, 2010. doi: 10.1111/j.1365-2966.2010.17328.x.

A. Pinzke, C. Pfrommer, and L. Bergstrom. Gamma-rays from dark matter annihilations strongly constrain the substructure in halos. Phys. Rev. Lett., 103:181302, 2009. doi: 10.1103/PhysRevLett.103.181302.

A. Pinzke, C. Pfrommer, and L. Bergstrom. Prospects of detecting gamma-ray emission from galaxy clusters: cosmic rays and dark matter annihilations. Phys. Rev., D84:123509, 2011. doi: 10.1103/PhysRevD.84.123509.

T. H. Reiprich and H. Boehringer. The mass function of an x-ray flux-limited sample of galaxy clusters. Nucl. Phys. Proc. Suppl., 80:0917, 2000.

M. Roos. Dark Matter: The evidence from astronomy, astrophysics and cosmology. ArXiv e-prints, Jan. 2010.

M. A. Sánchez-Conde, M. Cannoni, F. Zandanel, M. E. Gomez, and F. Prada. Dark matter searches with Cherenkov telescopes: nearby dwarf galaxies or local galaxy clusters? JCAP, 1112:011, 2011. doi: 10.1088/1475-7516/2011/12/011.

T. Sjöstrand, S. Ask, J. R. Christiansen, R. Corke, N. Desai, P. Ilten, S. Mrenna, S. Prestel, C. O. Rasmussen, and P. Z. Skands. An 
Introduction to PYTHIA 8.2. Comput. Phys. Commun., 191:159-177, 2015. doi: 10.1016/j.cpc.2015.01.024.

G. P. Smith et al. LoCuSS: Testing hydrostatic equilibrium in galaxy clusters. Mon. Not. Roy. Astron. Soc., 456(1):L74-L78, 2016. doi: $10.1093 / \mathrm{mnrasl} / \mathrm{slv} 175$.

M. Sun, D. Jerius, and C. Jones. A Small x-ray corona of the narrow-angle tail radio galaxy NGC 1265 soaring through the Perseus cluster. Astrophys. J., 633:165-173, 2005. doi: 10.1086/452620.

G. B. Taylor, N. E. Gugliucci, A. C. Fabian, J. S. Sanders, G. Gentile, and S. W. Allen. Magnetic fields in the center of the perseus cluster. Mon. Not. Roy. Astron. Soc., 368:1500-1506, 2006. doi: 10.1111/j.1365-2966.2006.10244.x.

H. Zhao. Analytical models for galactic nuclei. Mon. Not. Roy. Astron. Soc., 278:488-496, 1996. doi: 10.1093/mnras/278.2.488.

S. Zimmer, J. Conrad, and A. Pinzke. A Combined Analysis of Clusters of Galaxies - Gamma Ray Emission from Cosmic Rays and Dark Matter. 2011. 